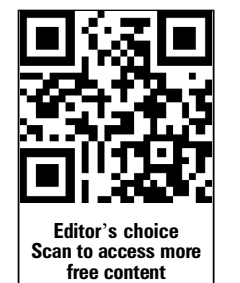

- Additional material is published online only. To view please visit the journal online (http://dx.doi.org/10.1136/ bjsports-2013-093302).

${ }^{1}$ British Athletics Medical Team, London, UK ${ }^{2}$ Department of Radiology, Royal Orthopaedic Hospital Foundation Trust Birmingham, UK ${ }^{3}$ Department of Radiology, Chelsea and Westminster Hospital, London, UK ${ }^{4}$ British Athletics Medical Team, British Athletics National Performance Centre, LoughboroughUniversity, Loughborough, UK

Correspondence to Dr Noel Pollock, British Athletics Medical Team, Level 1 Outpatients, Hospita of St John and St Elizabeth, 60 Grove End Road, London NW8 9NH, UK: npollock@uka.org.uk

Accepted 26 June 2014 Published Online First 16 July 2014
CrossMark

To cite: Pollock $\mathrm{N}$ James SLJ, Lee JC, et al. Br J Sports Med 2014:48: 1347-1351.

\title{
British athletics muscle injury classification: a new grading system
}

\author{
Noel Pollock, ${ }^{1}$ Steven L J James, ${ }^{2}$ Justin C Lee, ${ }^{3}$ Robin Chakraverty ${ }^{4}$
}

\section{ABSTRACT}

The commonly used muscle injury grading systems based on three grades of injury, representing minor, moderate and complete injuries to the muscle, are lacking in diagnostic accuracy and provide limited prognostic information to the clinician. In recent years, there have been a number of proposals for alternative grading systems. While there is recent evidence regarding the prognostic features of muscle injuries, this evidence has not often been incorporated into the grading proposals. The British Athletics Muscle Injury Classification proposes a new system, based on the available evidence, which should provide a sound diagnostic base for therapeutic decision-making and prognostication. Injuries are graded 0-4 based on MRI features, with Grades 1-4 including an additional suffix ' $a$ ', ' $b$ ' or ' $c$ ' if the injury is 'myofascial', 'musculo-tendinous' or 'intratendinous'. Retrospective and prospective studies in elite track and field athletes are underway to validate the classification for use in hamstring muscle injury management. It is intended that this grading system can provide a suitable diagnostic framework for enhanced clinical decisionmaking in the management of muscle injuries and assist with future research to inform the development of improved prevention and management strategies.

\section{INTRODUCTION}

Muscle injuries are common in sport and account for substantial time lost from training and competition. $^{1-3}$ They represented $48 \%$ of all injuries during track and field competition in a recent International Association of Athletics Federations (IAAF) study ${ }^{2}$ and more than $30 \%$ of all injuries in professional football. ${ }^{3}$ The hamstrings are the most frequently injured muscle group ${ }^{2}$ and hamstring muscle injuries alone result in an average of 90 days missed per club per season in professional soccer. ${ }^{4}$ Muscle injuries are also common in rugby union, ${ }^{5}{ }^{6}$ Australian Rules football, ${ }^{7}$ basketball ${ }^{8}$ and other Olympic sports. ${ }^{9}$

Grading systems are important for clinicians, coaches and athletes and should provide prognostic and therapeutic direction. The most widely used $^{10} 11$ current muscle grading systems are simple and, whether clinical or radiological, are usually based on three grades of injury which essentially represent minor, moderate and complete injuries to the muscle. ${ }^{12-15}$ Within these widely used grading systems there could be considered a lack of consistency in terminology and clarity in diagnostic entities. There is little representation of the current evidence that does provide prognostic information for the clinician such as length of muscle tear on MRI, ${ }^{10}{ }^{16}$ MRI 'negative' injuries, ${ }^{10}$ 17-19 distance from the origin, ${ }^{20}$ cross-sectional area of oedema $^{21} 22$ and involvement of the tendon. ${ }^{20} 2223$ There is some lack of consistency regarding the relevance of distance from the muscle origin, ${ }^{20-22}$ but the other features have repeatedly been demonstrated to have prognostic relevance.

In clinical practice as well as in recent literature $^{2425}$ the concept of the grade 0 muscle injury has been developed. This usually represents a clinical syndrome of muscle abnormality but without imaging evidence of pathology. It is of course possible, and indeed probable, that this is often a structural pathology, which is undetectable with current imaging modalities. ${ }^{26} 27$ Nevertheless, this 'nonstructural' injury grade has been associated with quicker return to sport $^{1011} 18$ and is therefore of relevance in a grading system.

Recently, the Munich consensus ${ }^{25}$ and others ${ }^{28}$ have proposed alternative muscle grading systems. The Munich grading system classifies injuries as either 'functional' (fatigue induced, delayed onset muscle soreness (DOMS), spine-related neuromuscular dysfunction or muscle-related neuromuscular dysfunction) or 'structural' muscle pathology. The 'functional' terminology is problematic as clearly a number of the pathological entities which the Munich system describes as 'functional' are most likely structural pathology, ${ }^{27}$ and indeed the consensus comments on MRI changes in these classes. The 'structural' classes are further divided into three essential grades of muscle injury: minor, moderate and complete. Clinical presentations of this pathology, categorised as 'structural' rather than 'functional', are likely to have important functional elements to the diagnosis and therefore this terminology has some limitations. ${ }^{29} 30$

A recent study by contributors to the Munich consensus concluded that, as with the previous grade 1-3 systems, the 'structural' part of the Munich categorisation was helpful prognostically but the 'functional' aspect of the system was not. ${ }^{31}$ However, the wide range of return to play durations in this study suggests that truly beneficial prognostic information from this grading system is still lacking. The Munich proposal neglects much of the recent evidence which demonstrates prognostic significance for site, length, tendon involvement, MRI negative presentations and cross-

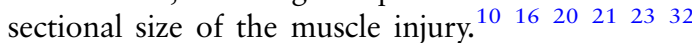
Therefore, particularly with regard to structural pathology, this system does not develop muscle injury grading substantially beyond the simplistic grade 1-3 systems currently in use which are really limited in their differentiation of injury, prognostic ability and therapeutic relevance. With regard to the 'functional' grading aspect of the Munich consensus, despite the lack of clearly defined diagnostic 
entities or evidence of prognosticating ability, this may still be of use to clinicians to develop understanding and to direct treatment strategies.

An alternative classification system proposed by Chan et al ${ }^{28}$ describes an imaging categorisation based on the location and type of tissue injury. There is undoubted merit in an anatomical diagnosis which may have prognostic relevance. However the nomenclature retains the poorly defined 'strain' term, does not clearly define the limits of each grade, ignores MRI negative injuries and the great majority of actual tears are still within the same grade. As both MRI resolution and our understanding of the relevant prognostic and anatomical features of muscle injuries improve, a grading system which uses these advances can be developed. The recent proposal by Chan et al and the Munich consensus are welcome additions that have stimulated interest in this area. However, the opportunity to provide a clinically relevant, evidentially coherent and logical approach remains.

The British Athletics Medical team provides medical support to Great Britain international track and field athletes. A recent report on injuries in 214 elite British track and field athletes over a 3.5-year time period (2010-2013) found that of the total 1000 injuries recorded, 147 (14.7\%) were hamstring-related injuries (unpublished). The grading of these injuries using the historical system demonstrated a broad prognostic value, similar to the recently published work in football. ${ }^{11}$ However, the demand of elite sport necessitates greater diagnostic accuracy with the goal of providing targeted management and rehabilitation within more clearly defined timescales. The British Athletics Medical team have therefore developed a muscle injury grading system that has a clear diagnostic framework and uses the available prognostic evidence to assist in classification. It has been primarily developed as a hamstring injury classification, influenced by the literature in this field, but with potential to be extrapolated for use in other muscle injuries.

\section{BRITISH ATHLETICS MUSCLE INJURY CLASSIFICATION Overview}

There are five grades of muscle injury categorised in this grading system: grade 0 through grade 4 , primarily, and exclusively for grades $1-4$, based on the MRI features of the muscle injury (see online supplementary table S1). There is clinical overlap between the grades but the most common clinical presentations are described as means of introduction. Grades 1-4 are further subcategorised into one of three diagnostic groups $(a, b$ or $c)$ based on the site and extent of the injury. The injury is classified at the highest number and letter as determined by the injury characteristics.

It is considered that muscle injury is an appropriate general term to encompass grade 0-4 injuries. In agreement with the Munich Consensus, ${ }^{25}$ the use of the term ' strain' is not recommended and it is more appropriate to use 'tear' to describe grade 1-4 injuries.

For grade 1-4 injuries, the suffix 'a' denotes a myofascial injury in the peripheral aspect of the muscle, ' $b$ ' an injury within the muscle belly, most commonly at the muscle tendon junction (MTJ) and 'c' an injury which extends into the tendon. The most common site of muscle injury is at the $\mathrm{MTJ}^{33}$ and this may be associated with more prolonged and different rehabilitation requirements than a peripheral myofascial injury. There is evidence that injury within the tendon is associated with a poorer prognosis ${ }^{16}{ }^{18}$ and therefore intratendon involvement has been categorised at the end of the scale as ' $c$ '.

There is an additional descriptor included in the classification to denote the site of injury (proximal, central or distal third) relative to the muscle origin. ${ }^{20}$ With respect to hamstring injuries, it is proposed that the proximal third is above the lower margin of the gluteus maximus and the distal third is below the origin of the short head of biceps femoris.

The specific muscle that has been injured should also be named (figure 1 ).

MRI

MRI may be performed on either a 1.5 or $3 \mathrm{~T}$ system, ideally at 24-48 h following injury. ${ }^{11}$ Skin markers (cod liver oil capsule) should be placed at the site of the athlete's maximum pain prior to imaging. The MRI study should include a combination of acquisitions in three orthogonal planes. The closest muscle insertion to the injury site should be included as this will define the proximal or distal extent of imaging. It is often necessary, particularly if the athlete's symptoms are poorly localised, to cover the whole thigh to ensure an optimal study. The exact choice of sequences will, to some extent, depend on the individual radiologist's preference. A typical protocol would include axial, coronal and sagittal short tau inversion recovery (STIR)/ T2-weighted fat suppressed/proton density-weighted fat suppressed sequences followed by axial and sagittal T1-weighted sequences. The coronal and sagittal sequences are primarily used to assess the longitudinal extent of the injury and tendon involvement, and the axial images allow optimal anatomical information and the cross-sectional area to be defined. The slice thickness of imaging acquisition should allow accurate definition of small injuries often necessitating a slice thickness of $4 \mathrm{~mm}$. This will often require the use of two separate axial acquisitions if the whole thigh is imaged for optimum resolution.

\section{Detailed description}

Grade 0 injuries

In the British Athletics Classification system grade 0 injuries are classified as: $0 \mathrm{a}-\mathrm{a}$ focal neuromuscular injury with normal MRI, or $0 \mathrm{~b}$ - generalised muscle soreness with normal MRI or MRI characteristic of DOMS. It is recognised that there may be clinical suspicion of a neural component to these grade 0 presentations $^{34}$ and this can be represented by the addition of ' $+\mathrm{N}$ ' to either of these injuries.

Grade 0 a classifies a clinical presentation of focal muscle soreness usually after exercise, although it may also occur during exercise. It is often accompanied with awareness on muscle contraction, but no or little inhibition of contraction or reduction in strength on manual testing. The clinician may be able to palpate a focal area of increased muscle tone. This clinical picture probably reflects a pathological process of microscopic muscle damage or peripheral nerve irritation. The grade $0 \mathrm{a}$ is an 'MRI-negative muscle injury', which is described in the literature and associated with improved prognosis. ${ }^{10}$ 17-19

Grade $0 \mathrm{~b}$ represents generalised muscle soreness, which most commonly occurs after unaccustomed exercise, often with an eccentric bias and is frequently termed DOMS. There may be characteristic MRI changes with generalised, patchy high signal change affecting several muscles.

\section{Grade 1-4 injuries}

Grade 1 injuries

Grade 1 injuries are small injuries (tears) to the muscle. The athlete will usually present with pain during or after activity. The athlete's range of movement at $24 \mathrm{~h}$ will usually be normal and although there may be pain on contraction, strength and initiation of contraction may be well maintained on clinical examination. 

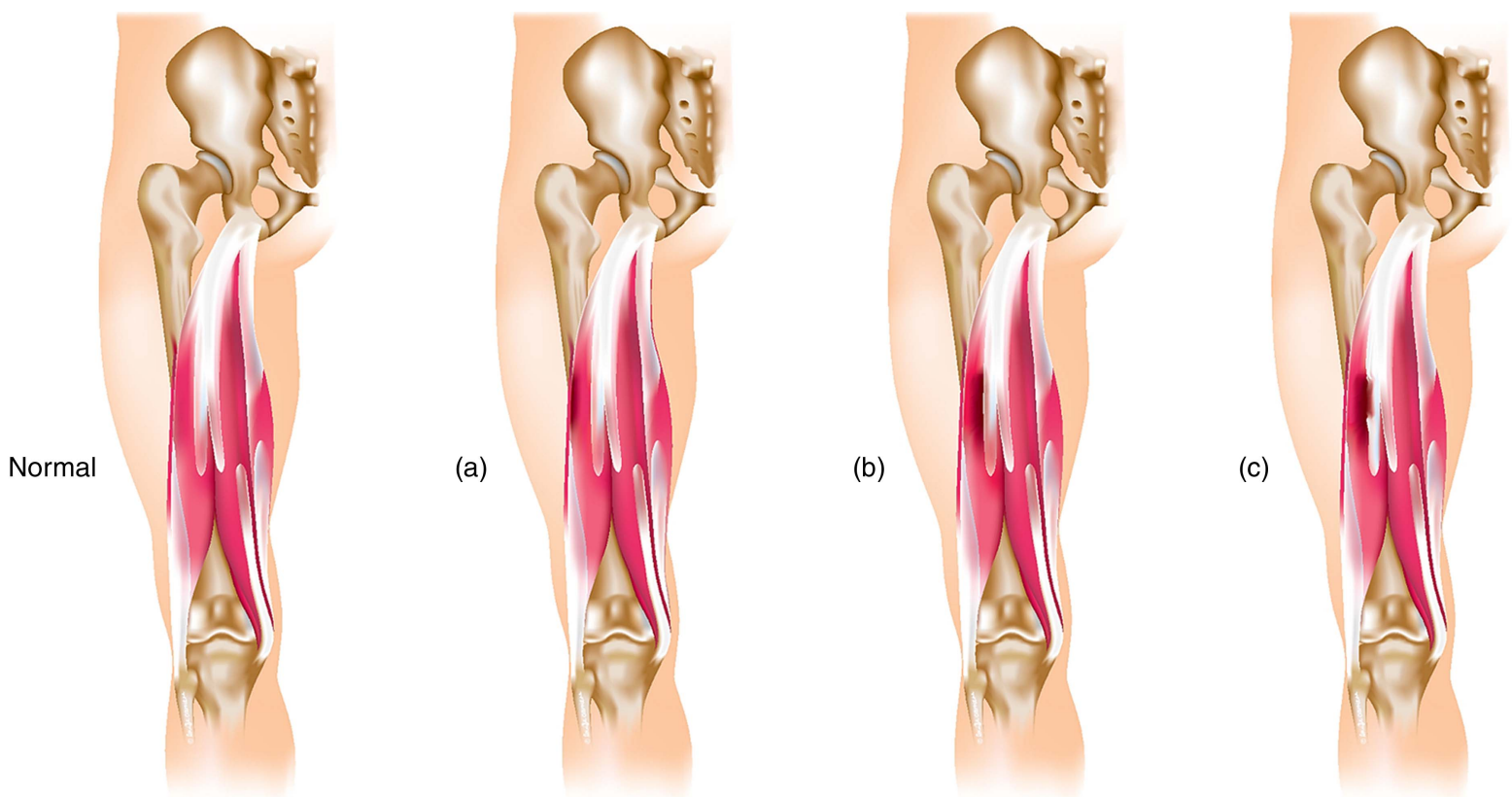

Figure 1 Letter classification dependent on anatomical site of muscle injury. (a) Myofascial, (b) musculo-tendinous, (c) intratendinous.

Grade 1a injuries extend from the fascia and demonstrate high signal change on fat suppressed/STIR images within the periphery of the muscle, no greater than $10 \%$ into the muscle and with a longitudinal length of less than $5 \mathrm{~cm}$ within the muscle. Frank muscle fibre disruption is not usually seen in this grade of injury but evidence of fibre disruption of less than $1 \mathrm{~cm}$ with limited high signal change, as noted above, may still be classified in this grade. Intermuscular fluid/haematoma on MRI may be evident within the fascial planes over a greater distance.

Grade $1 \mathrm{~b}$ injuries are sited within the muscle or, more commonly, at the MTJ. High signal change is evident at this site and extends over a limited area of less than $5 \mathrm{~cm}$ and less than $10 \%$ of the muscle cross-sectional area at its maximal site. Frank muscle fibre disruption is not usually seen in this grade of injury but evidence of fibre disruption of less than $1 \mathrm{~cm}$ with limited high signal change, as noted above, may still be classified in this grade.

As previously discussed, there is a poor prognostic significance of intratendinous extension and therefore there are no grade 1 injuries in this classification which involve disruption within the tendon (figure 2)..$^{20} 23$

\section{Grade 2 injuries}

Grade 2 injuries are moderate injuries (tears) to the muscle. The athlete will usually present with pain during activity which necessitates them to stop activity. The range of movement of the affected limb at $24 \mathrm{~h}$ will usually show some limitation with pain on initiation of contraction, usually with detectable weakness by the clinician.

Grade $2 \mathrm{a}$ injuries usually extend from the peripheral fascia into the muscle. Clinical experience suggests that they may be associated with a clinical history of pain during change of direction and manual strength testing may be less reduced with grade 2 a injuries relative to other grade 2 injuries. On MRI, high signal change will be evident from the periphery of the muscle. The high signal change will either measure between $10 \%$ and $50 \%$ of the cross-sectional area of that individual muscle at the site of injury or extend between 5 and $15 \mathrm{~cm}$ within the muscle. Architectural fibre disruption will be less than $5 \mathrm{~cm} .{ }^{16}$
Grade $2 \mathrm{~b}$ injuries occur within the muscle or, more commonly, at the MTJ. On MRI, the high signal change will either measure between $10 \%$ and $50 \%$ of the muscle cross-sectional area $^{35} 36$ or have a longitudinal length between 5 and $15 \mathrm{~cm}$. There is likely to be evidence of muscle fibre disruption of less than $5 \mathrm{~cm}^{16}$

Grade $2 c$ injuries extend into the tendon but injury within the tendon is evident over a longitudinal length of less than $5 \mathrm{~cm}$ and less than $50 \%$ of the maximal tendon diameter on axial images. If the injury is near the end of the free tendon there may be some loss of tension in the free tendon. It may still be classified as a $2 c$, rather than $3 c$, if the injury size is compatible with the measurements above (figures 3-5).

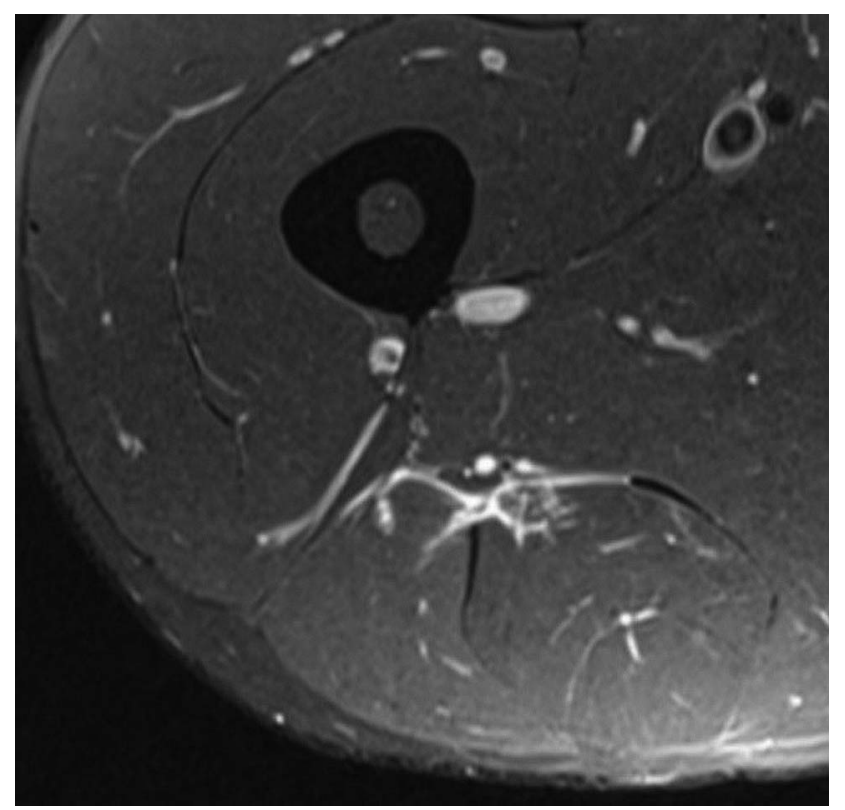

Figure 2 Grade $1 \mathrm{~b}$ injury to long head of biceps femoris. 


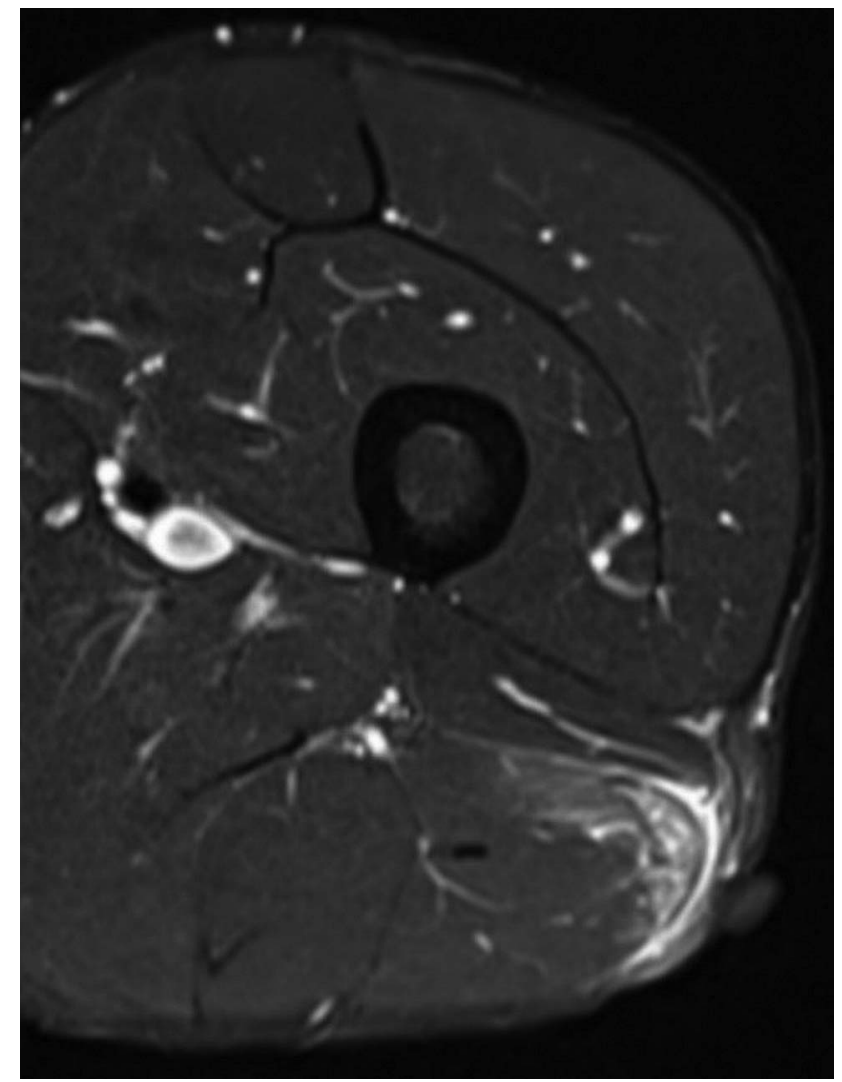

Figure 3 Grade 2a injury to lateral aspect of long head of biceps femoris.

\section{Grade 3 injuries}

Grade 3 injuries are extensive tears to the muscle. The athlete will usually present with sudden onset pain and may fall to the ground. Their range of movement at $24 \mathrm{~h}$ is usually significantly reduced with pain on walking. There is usually obvious weakness in contraction.

Grades 3a (myofascial) and 3b (muscular/musculotendinous) will demonstrate MRI features of high signal change patterns of greater than $50 \%$ of the muscle cross-sectional area or greater

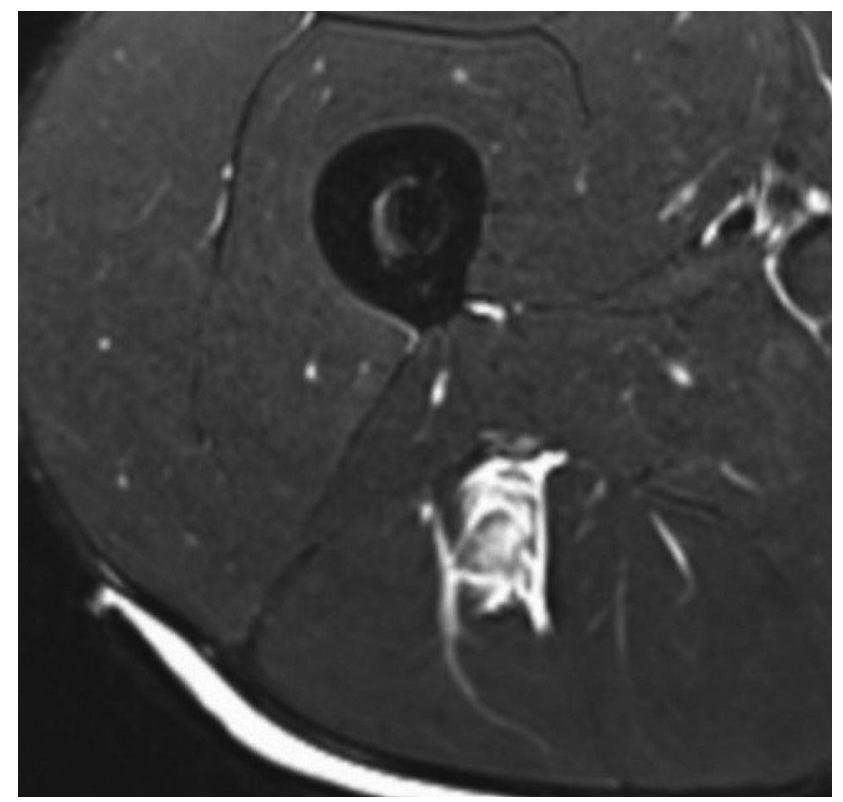

Figure 4 Grade 2b injury to long head of biceps femoris.

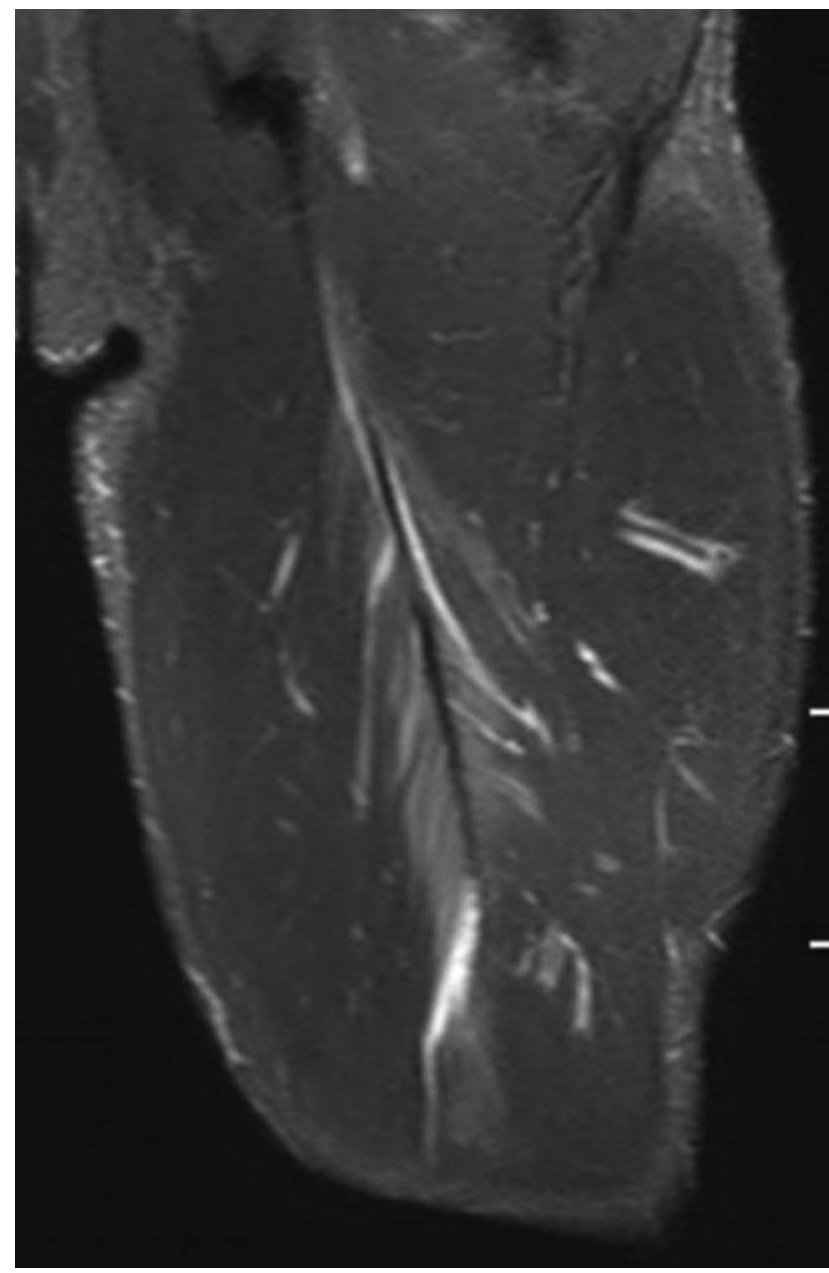

Figure 5 Grade 2c injury to long head of biceps femoris.

than $15 \mathrm{~cm}$ in length. There will be evidence of architectural fibre disruption which is likely to be greater than $5 \mathrm{~cm}$. Grades $3 \mathrm{a}$ and $3 \mathrm{~b}$ are differentiated by the location extending to the periphery (3a) or being within the muscle/at the MTJ (3b).

Grade 3c (intratendinous) injuries have evidence of injury in the tendon over a longitudinal length of greater than $5 \mathrm{~cm}$ or greater than $50 \%$ of the tendon's maximal cross-sectional area. There is no evidence of a complete defect but there may be loss of the usual straight margins and tendon tension suggesting some loss of the tendon integrity (figure 6).

\section{Grade 4 injuries}

Grade 4 injuries are complete tears to either the muscle (grade 4) or tendon (grade 4c). The athlete will experience sudden onset pain and significant and immediate limitation to activity. A palpable gap will often be felt. There may be less pain on contraction than with a grade 3 injury (figure 7 ).

\section{SUMMARY, LIMITATIONS AND FUTURE DIRECTION}

We propose a new muscle grading system that is anatomically focused and uses available evidence on prognostication of muscle injuries to inform the classification. It provides the clinician with relevant anatomical and pathological information which can be used to guide appropriate rehabilitation for the injured tissue. This could provide the basis for advice and appropriate communication to athletes and coaches, and also a structure for future research in the prevention and treatment of muscle injury in 


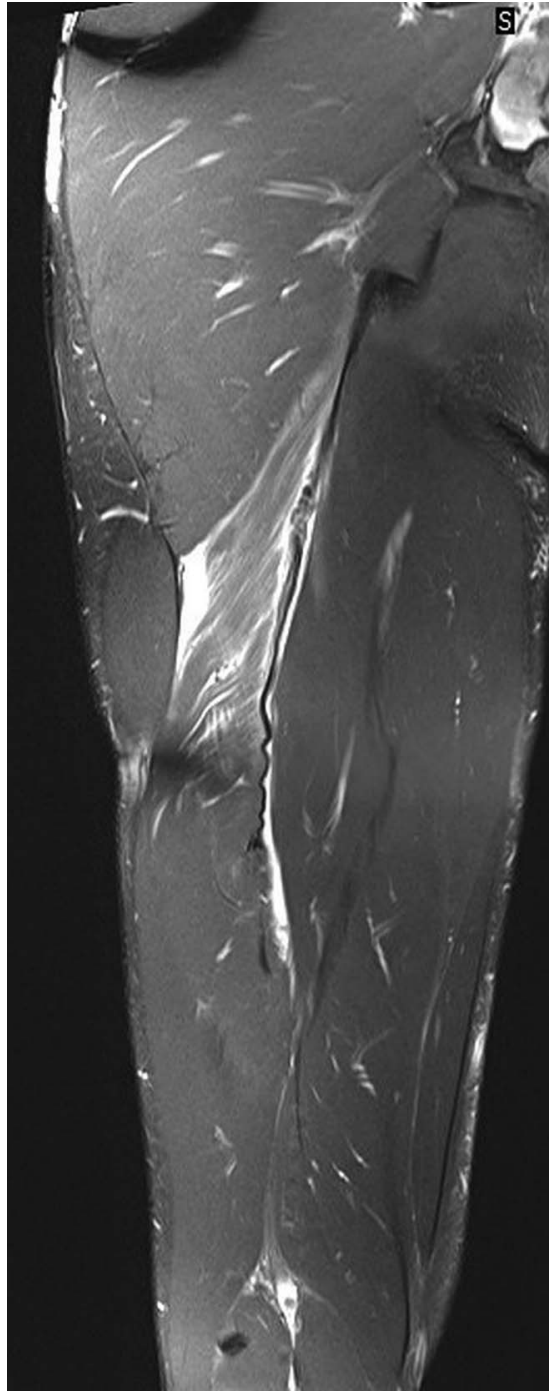

Figure 6 Grade 3c injury to long head of biceps femoris.

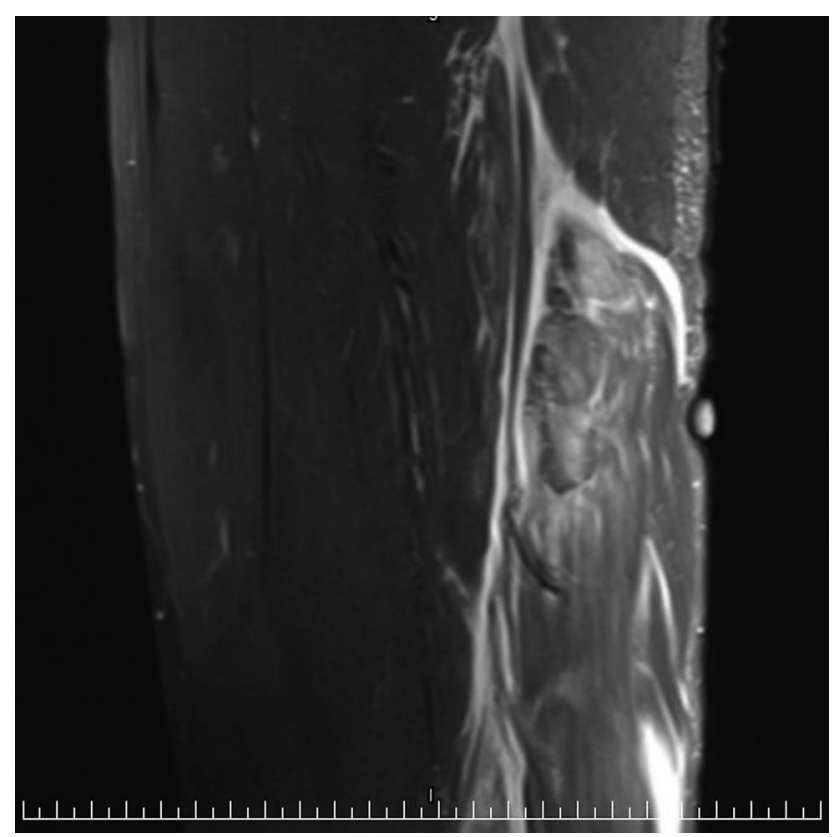

Figure 7 Grade 4 Injury to proximal biceps femoris. sport. With further development, it will be feasible to incorporate some relevant clinical prognostic signs into this grading system. These could be sport specific and, for example, may result in a ' + ' or ' - ' that is added to the grade based on the presence or absence of key clinical prognostic signs.

Musculoskeletal ultrasound is being increasingly used by clinicians and radiologists in the assessment of muscle injury. This is a subjective imaging modality that is dependent on the operator and, as such, it is more difficult to define and standardise ultrasound findings for a new classification system. The incorporation of ultrasound imaging into this classification system should be considered an area for future study.

It should be recognised that while this proposal is informed by the available evidence it is currently expert opinion and awaits validation. It is critical for muscle grading systems to be reproducible with excellent intraobserver and interobserver reliability. The clear diagnostic criteria in this grading system should assist in this regard. MRI has excellent interobserver and intraobserver reliability in acute hamstring injuries ${ }^{32}$ but it does not provide reliable information on player readiness to return to play. ${ }^{37}$ It is intended that this classification system can provide a reproducible diagnostic framework for enhanced clinical management of muscle injury in our sport and for future research.

\section{What are the new findings?}

A new muscle injury classification.

- Classification based on extent (grades $0-4)$ and site (a, b or c) of injury.

- Site of injury is determined as myofascial (a), muscularl musculotendinous (b) or intratendinous (c).

- Extent of injury is determined by MRI features of the muscle injury.

\section{How might it impact on clinical practice in the near future?}

- The classification system is in current use within elite track and field in the UK to provide validation for future clinical and research use.

- As this system has an ease of use, reproducibility and a clinically relevant and logical structure, it could have significant impact on enhancing current clinical practice.

Acknowledgements The authors acknowledge the support and advice of colleagues in the British Athletics medical team in the development of this grading system including Toby Smith, John Rogers, Jarrod Antflick, Ben MacDonald, Leigh Halfteck and Shane Kelly. The authors also acknowledge Louise Carrier for the illustrative work.

Contributors NP contributed to conceptual design and development of grading system, he was also manuscript author and provided clinical sports medicine expertise. SJ contributed to the refinement of imaging specifics of the grading system, drafting and development of concepts and provided radiological specific expertise. $J$ contributed to the refinement of imaging specifics of the grading system and provided radiological specific expertise. RCcontributed to the development and refinement of the grading system, drafting of the article and provided clinical sports medicine expertise.

Provenance and peer review Not commissioned; externally peer reviewed. 


\section{REFERENCES}

1 Freckleton $G$, Pizzari T. Risk factors for hamstring muscle strain injury in sport: a systematic review and meta-analysis. Br J Sports Med 2013;47:351-8.

2 Alonso J-M, Edouard P, Fischetto $G$, et al. Determination of future prevention strategies in elite track and field: analysis of Daegu 2011 IAAF Championships injuries and illnesses surveillance. Br J Sports Med 2012;46:505-14.

3 Ekstrand J, Hägglund $M$, Waldén $M$. Epidemiology of muscle injuries in professional football (soccer). Am J Sports Med 2011;39:1226-32.

4 Woods C, Hawkins RD, Maltby S, et al. The Football Association Medical Research Programme: an audit of injuries in professional football-analysis of hamstring injuries. Br J Sports Med 2004;38:36-41.

5 Brooks JHM, Fuller CW, Kemp SPT, et al. Epidemiology of injuries in English professional rugby union: part 2 training injuries. Br J Sports Med 2005;39: 767-75.

6 Brooks JHM, Fuller CW, Kemp SPT, et al. Epidemiology of injuries in English professional rugby union: part 1 match injuries. Br J Sports Med 2005;39:757-66.

7 Orchard J, Seward H. Epidemiology of injuries in the Australian Football League, seasons 1997-2000. Br J Sports Med 2002;36:39-44.

8 Meeuwisse $W H$, Sellmer $R$, Hagel BE. Rates and risks of injury during intercollegiate basketball. Am J Sports Med 2003;31:379-85.

9 Engebretsen L, Soligard T, Steffen $\mathrm{K}$, et al. Sports injuries and illnesses during the London Summer Olympic Games 2012. Br J Sports Med 2013;47:407-14.

10 Kerkhoffs GMMJ, van Es N, Wieldraaijer T, et al. Diagnosis and prognosis of acute hamstring injuries in athletes. Knee Surg Sports Traumatol Arthrosc 2013;21:500-9.

11 Ekstrand J, Healy JC, Walden $\mathrm{M}$, et al. Hamstring muscle injuries in professional football: the correlation of MRI findings with return to play. Br J Sports Med 2011:46:112-17.

12 Brandser EA, el-Khoury GY, Kathol MH, et al. Hamstring injuries: radiographic, conventional tomographic, CT, and MR imaging characteristics. Radiology 1995; 197:257-62.

13 Takebayashi $\mathrm{S}$, Takasawa $\mathrm{H}$, Banzai $\mathrm{Y}$, et al. Sonographic findings in muscle strain injury: clinical and MR imaging correlation. J Ultrasound Med 1995;14:899-905.

14 Peetrons P. Ultrasound of muscles. Eur Radiol 2002;12:35-43.

15 O'Donoghue DH. Treatment of injuries to athletes. Saunders, 1984:742.

16 Connell DA, Schneider-Kolsky ME, Hoving JL, et al. Longitudinal study comparing sonographic and MRI assessments of acute and healing hamstring injuries. AJR Am J Roentgenol 2004;183:975-84.

17 Ekstrand J, Healy JC, Waldén $\mathrm{M}$, et al. Hamstring muscle injuries in professional football: the correlation of MRI findings with return to play. Br J Sports Med 2012:46:112-17.

18 Cross TM, Gibbs N, Houang MT, et al. Acute quadriceps muscle strains: magnetic resonance imaging features and prognosis. Am J Sports Med 2004;32:710-19.

19 Gibbs NJ, Cross TM, Cameron M, et al. The accuracy of MRI in predicting recovery and recurrence of acute grade one hamstring muscle strains within the same season in Australian Rules football players. J Sci Med Sport 2004;7:248-58.
20 Askling CM, Tengvar M, Saartok T, et al. Acute first-time hamstring strains during high-speed running: a longitudinal study including clinical and magnetic resonance imaging findings. Am J Sports Med 2006;35:197-206.

21 Slavotinek J. Muscle injury: the role of imaging in prognostic assignment and monitoring of muscle repair. Semin Musculoskelet Radiol 2010;14:194-200.

22 Cohen SB, Towers JD, Zoga A, et al. Hamstring injuries in professional football players: magnetic resonance imaging correlation with return to play. Sports Health 2011;3:423-30.

23 Comin J, Malliaras $\mathrm{P}$, Baquie $\mathrm{P}$, et al. Return to competitive play after hamstring injuries involving disruption of the central tendon. Am J Sports Med 2013:41:111-15.

24 Stoller DW. Magnetic resonance imaging in orthopaedics and sports medicine. Lippincott Williams \& Wilkins, 2007:1112.

25 Mueller-Wohlfahrt H-W, Haensel L, Mithoefer K, et al. Terminology and classification of muscle injuries in sport: the Munich consensus statement. $\mathrm{Br} J$ Sports Med 2013;47:342-50.

26 Meyer RA, Prior BM. Functional magnetic resonance imaging of muscle. Exerc Sport Sci Rev 2000;28:89-92.

27 Cermak NM, Noseworthy MD, Bourgeois JM, et al. Diffusion tensor MRI to assess skeletal muscle disruption following eccentric exercise. Muscle Nerve 2012;46:42-50.

28 Chan 0, Del Buono A, Best TM, et al. Acute muscle strain injuries: a proposed new classification system. Knee Surg Sports Traumatol Arthrosc 2012;20:2356-62.

29 Fousekis $\mathrm{K}$, Tsepis $\mathrm{E}$, Poulmedis $\mathrm{P}$, et al. Intrinsic risk factors of non-contact quadriceps and hamstring strains in soccer: a prospective study of 100 professional players. Br J Sports Med 2010;45:709-14.

30 Fyfe JJ, Opar DA, Williams MD, et al. The role of neuromuscular inhibition in hamstring strain injury recurrence. J Electromyogr Kinesiol 2013;23:523-30.

31 Ekstrand J, Askling C, Magnusson $\mathrm{H}$, et al. Return to play after thigh muscle injury in elite football players: implementation and validation of the Munich muscle injury classification. Br J Sports Med 2013;47:769-74

32 Hamilton B, Whiteley R, Almusa $\mathrm{E}$, et al. Excellent reliability for MRI grading and prognostic parameters in acute hamstring injuries. Br J Sports Med 2014:48:1385-7.

33 El-Khoury GY, Brandser EA, Kathol MH, et al. Imaging of muscle injuries. Skeletal Radiol 1996:25:3-11.

34 Orchard JW. Lumbar spine region pathology and hamstring and calf injuries in athletes: is there a connection? Br J Sports Med 2004;38:502-4.

35 Slavotinek JP, Verrall GM, Fon GT. Hamstring injury in athletes: using MR imaging measurements to compare extent of muscle injury with amount of time lost from competition. AJR Am J Roentgenol 2002;179:1621-8.

36 Pomeranz SJ, Heidt RS Jr. MR imaging in the prognostication of hamstring injury. Radiology 1993;189:897-900.

37 Reurink G, Goudswaard GJ, Tol JL, et al. MRI observations at return to play of clinically recovered hamstring injuries. Br J Sports Med 2014:48:1370-6. 